\title{
New data on the occurrence of longhorn beetles (Coleoptera: Cerambycidae) in the Eastern Beskid Mountains (Poland)
}

\author{
Lech KARPIŃSKI, Artur TASZAKOWSKI and Wojciech T. SZCZEPAŃSKI \\ Department of Zoology, Faculty of Biology and Environmental Protection, \\ University of Silesia, Bankowa 9, 40-007 Katowice, Poland; \\ e-mails: lkarpinski@us.edu.pl (corresponding author); ataszakowski@us.edu.pl; wszczepanski@us.edu.pl
}

\begin{abstract}
New data on the occurrence of the 70 species of longhorn beetles from the area of the Eastern Beskid Mountains including the Low Beskids (56 species) are presented. The state of knowledge about Cerambycidae of the Eastern Beskid Mountains, Low Beskids, Western Beskid Mountains and Bieszczady Mountains is summarised. New localities of some rarely seen species as: Pachyta quadrimaculata (Linnaeus 1758), Stenocorus meridianus (Linnaeus, 1758), Evodinus clathratus (Fabricius, 1793), Anoplodera rufipes (Schaller, 1783), A. sexguttata (Fabricius, 1775), Ropalopus macropus (Germar, 1824), Pogonocherus hispidus (Linnaeus, 1758), Phytoecia cylindrica (Linnaeus 1758) and Ph. nigricornis (Fabricius 1782) are given. Four species, P. quadrimaculata, Leptura annularis Fabricius, 1801, Pyrrhidium sanguineum (Linnaeus, 1758) and Pogonocherus fasciculatus (DeGeer, 1775), are recorded for the first time from the area of the Low Beskids.
\end{abstract}

Key words: cerambycids, the Low Beskids, rare species, new localities, Carpathian fauna

\section{INTRODUCTION}

The longhorn beetle family (Cerambycidae) is one of the group of beetles (Coleoptera) that is richest in species with approximately 35000 described species (Švácha \& Lawrence 2014), and 192 of these species occur in Poland (Gutowski et al. 2012). Despite the numerous works that have been devoted to cerambycids, the knowledge on their distribution in Poland is still insufficient, and some regions of the country require further studies (Zieliński 2004, Gutowski et al. 2011). The occurrence of longhorn beetles in southeastern Poland is fairly well known thanks mainly to papers by Trella (1925) and Gutowski (1995) on the Eastern Beskid Mountains and thanks to the works by Kubisz \& Hilszczański (1992) and Olbracht \& Szewkienicz (2013) on the Low Beskids. The present study provides some new data on many species of cerambycids in the part of the Eastern Beskid Mountains, where detailed research have not been conducted so far. The work aims to supplement the knowledge on the longhorn beetles diversity of the Eastern Beskid Mountains.

\section{AREA AND METHODS}

According to the division of Poland used in the Catalogue of Fauna of Poland (Burakowski et al. 1990) the research area is located in the Eastern Beskid Mountains (Beskid Wschodni). However, according to physico-geographical regionalisation of Poland proposed by Kondracki (2013), this area is located in two macroregions. The first is called the Foothills of the Central Beskids (Pogórze Środkowobeskidzkie) and it consists with eight mesoregions. Only two of them were sampled here, i.e. the Gorlice Depression (Obniżenie Gorlickie) and the Jasło Foothills (Pogórze Jasielskie). The second macroregion with our plots is the region of the Central Beskids (Beskidy Środkowe) which includes only one mesoregion - the Low Beskids (Beskid Niski). In this paper we keep the names of regions used in the Catalogue of Fauna of 
Poland referring our data to Eastern Beskid Mountains, but additionaly we distinguished in it the area of the Low Beskids. In this way we want to highlight the specificity of the fauna of the region with its relatively low altitude and many mountain passes. Those passes are not very significant for species migration (Mazur 2001), but according to Taszakowski (2012), they can be considered to be migration routes for many species from the Pannonian Basin.

According to Kondracki (2013) the Low Beskids is a mountain range which forms a transition zone between the Eastern and Western Beskids. The Low Beskids is characterized by the occurrence of the so-called trans-Carpathian passes, which is associated with the lack or limited presence of plant species characteristic for the Carpathians, such as spruce Picea abies (L.) Karst. Because the average height of these mountains does not exceed $700 \mathrm{~m}$ above sea level, there are no plant communities that are characteristic for the higher parts of the mountain here (Kubisz \& Hilszczański 1992).

The research plots (in eight squares of the UTM grid) are in the following locations (in alphabetical order) (Fig. 1): EA20 - Bednarka, EV29 - Dobrynia, EA10 - Libusza, EA20 Lipinki, EV39 - Nowy Żmigród, EV39 - Wola Dębowiecka and within the Low Beskids: EV28 - Bartne, EV29 - Bednarka, EV17 - Blechnarka, EV18 - Gładyszów, EV18 Małastów 1, EV19 - Małastów 2, EV39 - Mrukowa, EV29 - Wapienne and EV17 - WysowaZdrój.

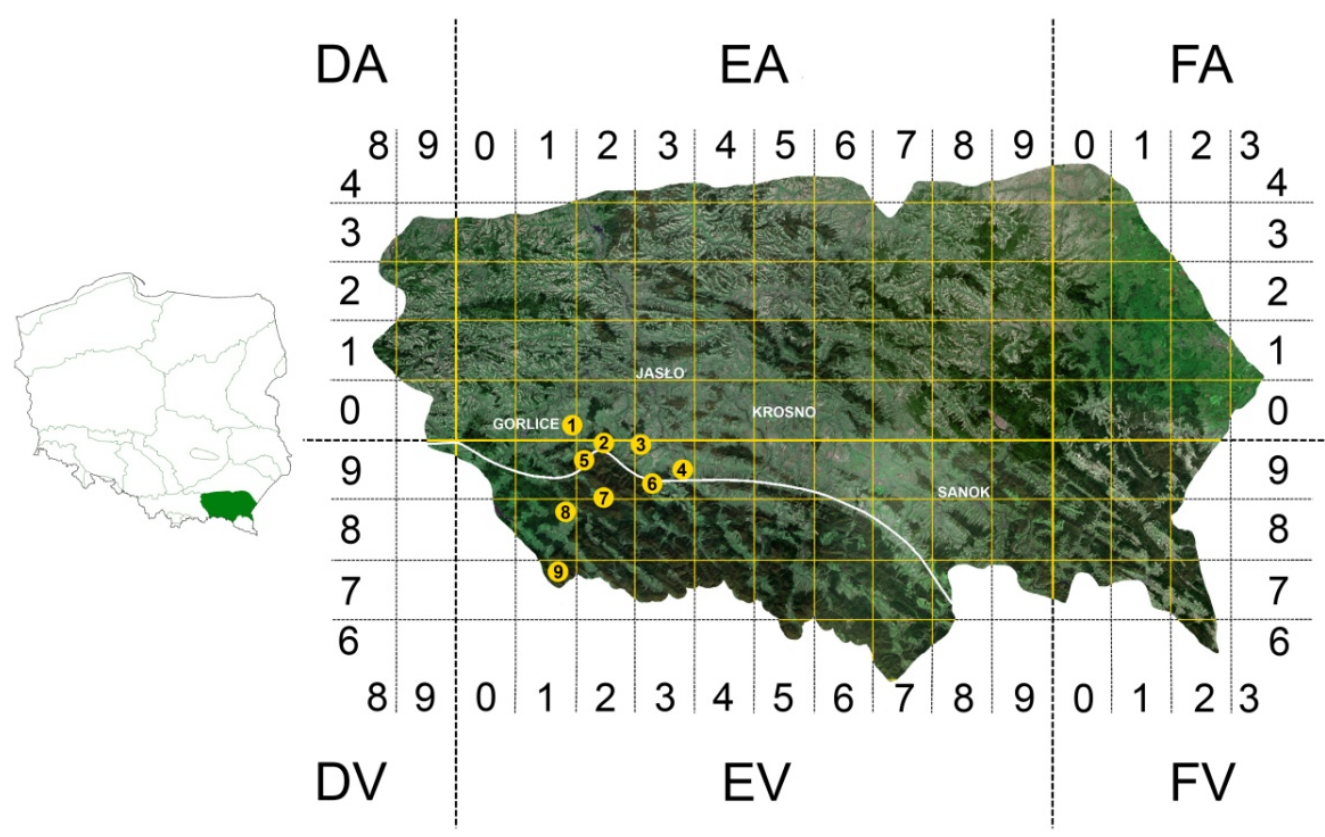

Fig. 1. The research plots within the Eastern Beskid Mountains: 1 - Libusza and Lipinki, 2 - Bednarka, 3 - Wola Dębowiecka and Dobrynia, 4 - Nowy Żmigród, 5 - Wapienne, 6 - Mrukowa, 7 - Bartne, 8 - Gładyszów and Małastów, 9 - Wysowa-Zrój and Blechnarka; white line - border of the Low Beskids. The map of Poland was created based on gis.biomap.pl. 
The studies were carried out between 2004-2014 using the methods of sighting of imagines and sweep-netting (grasslands and ecotone zones). Moreover, in the last three years, the research were conducted more intensely and uniformly using additional methods: shaking beetles down from flowering trees and shrubs into an entomological umbrella, and analyzing the feeding grounds in inhabited woods. Beetles were caught in different plant communities, e.g., the Carpathian beech forest and its bordering scrub (mainly in the Low Beskids), hornbeam forests (Bednarka), subxerothermic plant communities (Dobrynia) or from slowly overgrowing meadows and pastures.

The nomenclature was adopted from the Catalogue of Palearctic Coleoptera (Löbl \& Smetana 2010). The specimens are preserved in the collections of the authors (mainly in A. Taszakowski's collection).

\section{RESULTS}

During the study, the presence of 70 species of Cerambycidae was recorded (Table 1). The species represent about 36\% of the Polish fauna. In the Low Beskids 56 species were observed. Among them, four have been recorded in this region for the first time: Pachyta quadrimaculata (Linnaeus, 1758), Leptura annularis Fabricius, 1801, Pyrrhidium sanguineum (Linnaeus, 1758) and Pogonocherus fasciculatus (DeGeer, 1775).

Table 1. Longhorn beetles (Coleoptera: Cerambycidae) that were collected and/or observed during the study in the Eastern Beskid Mountains (with details of the Low Beskids). ! - species protected by law; * - species rarely observed in this part of the country; ${ }^{1}$ - species collected or observed during our research on the territory of the Low Beskids.

\begin{tabular}{|c|c|c|c|c|}
\hline No. & Species & Locality & Date & $\begin{array}{l}\text { Number of } \\
\text { specimens }\end{array}$ \\
\hline
\end{tabular}

\section{Prioninae Fairmaire, 1864}

1. Prionus coriarius (Linnaeus, 1758) ${ }^{1}$

\section{Lepturinae Latreille, 1802}

2. Oxymirus cursor (Linnaeus, 1758) ${ }^{1}$

3. Rhagium inquisitor (Linnaeus, 1758) ${ }^{1}$

4. Rhagium mordax (DeGeer, 1775) ${ }^{1}$

5. * Stenocorus meridianus (Linnaeus, 1758)

6. * Pachyta quadrimaculata (Linnaeus, 1758) ${ }^{1}$

7. ${ }^{*}$ Evodinus clathratus (Fabricius, 1793) ${ }^{1}$

8. Gaurotes virginea (Linnaeus, 1758) ${ }^{1}$

9. Dinoptera collaris (Linnaeus, 1758) ${ }^{1}$
EV17: Libusza

EV17: Blechnarka

EA10: Libusza EV17: Wysowa-Zdrój

EA10: Libusza EV17: Wysowa-Zdrój

EV17: Blechnarka EV17: Wysowa-Zdrój

EV17: Wysowa-Zdrój

EV17: Blechnarka

EV17: Blechnarka

EV17: Blechnarka EA10: Libusza

EA20: Lipinki EV17: Wysowa-Zdrój
16 Jun 2005

7 Jul 2008

3 exx.

17 Jun 2006

17 May 2013

4 exx.

8 Aug 2007

22 Jul 2005

2 May 2014

11 Jul 2006

2 Jul 2007

2 May 2014

27 Jun 2005

4 Jul 2006

20 May 2014

18 Jul 2007

8 Jul 2007

22 Jul 2008

13 Jun 2011

13 Jun 2011

27 Jun 2005

17 Jun 2007

12 Jun 2013

15 Jun 2013

21 May 2014
5 exx.

3 exx.

3 exx.

1 ex.

1 ex.

2 exx.

6 exx. 


\begin{tabular}{|c|c|c|c|}
\hline Species & Locality & Date & $\begin{array}{l}\text { Number of } \\
\text { specimens }\end{array}$ \\
\hline 10. $*$ Nivellia sanguinosa (Gyllenhal, 1827) ${ }^{1}$ & EV17: Wysowa-Zdrój & $\begin{array}{l}16 \text { May } 2013 \\
1 \text { May 2014: pupae } \\
\text { and imagines still } \\
\text { inside wood of dead } \\
\text { Padus avium }\end{array}$ & 6 exx. \\
\hline 11. Pidonia lurida (Fabricius, 1793) ${ }^{1}$ & $\begin{array}{l}\text { EV29: Bednarka } \\
\text { EV17: Wysowa-Zdrój }\end{array}$ & $\begin{array}{l}\text { 14 Jun } 2006 \\
\text { 7-8 Jul } 2010 \\
13 \text { Jun } 2011\end{array}$ & 4 exx. \\
\hline 12. Grammoptera ruficornis (Fabricius, 1781) & $\begin{array}{l}\text { EA20: Bednarka } \\
\text { EA10: Libusza } \\
\text { EV39: Nowy Żmigród }\end{array}$ & $\begin{array}{l}23 \text { May } 2014 \\
17 \text { Jun } 2006 \\
16 \text { Jun } 2013 \\
20 \text { May } 2014 \\
\text { 19 May } 2013\end{array}$ & 9 exx. \\
\hline 13. Alosterna tabacicolor (DeGeer, 1775) ${ }^{1}$ & $\begin{array}{l}\text { EA20: Lipinki } \\
\text { EV17: Wysowa-Zdrój }\end{array}$ & $\begin{array}{l}15 \text { Jun } 2013 \\
23 \text { Jul } 2010\end{array}$ & 2 exx. \\
\hline 14. * Anoplodera rufipes (Schaller, 1783) & EA20: Bednarka & 23 May 2014 & $1 \mathrm{ex}$. \\
\hline 15. * Anoplodera sexguttata (Fabricius, 1775) & EA10: Libusza & 20 Jun 2008 & $1 \mathrm{ex}$. \\
\hline 16. Pseudovadonia livida (Fabricius, 1777$)^{1}$ & $\begin{array}{l}\text { EV18: Gładyszów } \\
\text { EA10: Libusza } \\
\text { EV39: Mrukowa }\end{array}$ & $\begin{array}{l}\text { 14 Jun } 2013 \\
4 \text { Aug } 2005 \\
\text { 10 Jun } 2013 \\
\text { 13 Jun } 2013\end{array}$ & 9 exx. \\
\hline 17. Stictoleptura scutellata (Fabricius, 1781) ${ }^{1}$ & $\begin{array}{l}\text { EV28: Bartne } \\
\text { EV29: Bednarka } \\
\text { EV19: Małastów } 2 \\
\text { EV17: Wysowa-Zdrój }\end{array}$ & $\begin{array}{l}9 \text { Jul } 2013 \\
14 \text { Jun } 2006 \\
\text { 23 Jul } 2013 \\
\text { 14 Jun } 2013 \\
\text { 12 Jul } 2005\end{array}$ & 8 exx. \\
\hline 18. Stictoleptura maculicornis (DeGeer, 1775) ${ }^{\mathbf{1}}$ & $\begin{array}{l}\text { EV17: Blechnarka } \\
\text { EV17: Wysowa-Zdrój }\end{array}$ & $\begin{array}{l}\text { 13 Jun } 2011 \\
8 \text { Jul } 2010 \\
13 \text { Jun } 2011\end{array}$ & 6 exx. \\
\hline 19. Stictoleptura rubra (Linnaeus, 1858) ${ }^{1}$ & $\begin{array}{l}\text { EV29: Bednarka } \\
\text { EV17: Blechnarka } \\
\text { EV17: Wysowa-Zdrój }\end{array}$ & $\begin{array}{l}4 \text { Aug } 2009 \\
17 \text { Jul } 2007 \\
20 \text { Aug } 2009 \\
20 \text { Aug } 2010 \\
\text { 3-11 Aug } 2011\end{array}$ & 7 exx. \\
\hline 20. Stictoleptura tesserula (Charpentier, 1825) ${ }^{\mathbf{1}}$ & $\begin{array}{l}\text { EV28: Bartne } \\
\text { EA20: Bednarka }\end{array}$ & $\begin{array}{l}9 \text { Jul } 2013 \\
14 \text { Jun } 2006 \\
4 \text { Aug } 2009\end{array}$ & 8 exx. \\
\hline 21. Anastrangalia dubia $(\text { Scopoli, } 1763)^{1}$ & $\begin{array}{l}\text { EV28: Bartne } \\
\text { EV17: Blechnarka } \\
\text { EV17: Wysowa-Zdrój }\end{array}$ & $\begin{array}{l}9 \text { Jul } 2013 \\
\text { 13 Jun } 2011 \\
12 \text { Aug } 2006\end{array}$ & 4 exx. \\
\hline 22. Anastrangalia sanguinolenta (Linnaeus, 1761$)^{1}$ & $\begin{array}{l}\text { EA20: Bednarka } \\
\text { EV17: Blechnarka } \\
\text { EV17: Wysowa-Zdrój }\end{array}$ & $\begin{array}{l}\text { 14 Jun } 2006 \\
\text { 8 Jul } 2010 \\
\text { 8-23 Jul } 2010\end{array}$ & 4 exx. \\
\hline 23. Pachytodes cerambyciformis (Schrank, 1781) ${ }^{\mathbf{1}}$ & $\begin{array}{l}\text { EA20: Bednarka } \\
\text { EV17: Blechnarka } \\
\text { EA10: Libusza } \\
\text { EV17: Wysowa-Zdrój }\end{array}$ & $\begin{array}{l}9 \text { Jul } 2013 \\
13 \text { Jun } 2011 \\
4 \text { Aug } 2005 \\
20 \text { Aug } 2010\end{array}$ & 5 exx. \\
\hline 24. Leptura annularis Fabricius, $1801^{1}$ & $\begin{array}{l}\text { EV17: Blechnarka } \\
\text { EA10: Libusza }\end{array}$ & $\begin{array}{l}24 \text { Jul } 2013 \\
\text { 17 Jun } 2006\end{array}$ & 2 exx. \\
\hline
\end{tabular}




\begin{tabular}{|c|c|c|c|}
\hline Species & Locality & Date & $\begin{array}{l}\text { Number o } \\
\text { specimen }\end{array}$ \\
\hline 25. Leptura quadrifasciata Linnaeus, $1758^{1}$ & $\begin{array}{l}\text { EA10: Libusza } \\
\text { EV17: Wysowa-Zdrój }\end{array}$ & $\begin{array}{l}26 \text { Jun } 2012 \\
20 \text { Aug } 2006 \\
19 \text { Aug } 2009 \\
23 \text { Jul } 2010 \\
20 \text { Aug } 2010\end{array}$ & 5 exx. \\
\hline 26. Rutpela maculata (Poda, 1761$)^{1}$ & $\begin{array}{l}\text { EV29: Bednarka } \\
\text { EA10: Libusza } \\
\text { EV17: Wysowa-Zdrój }\end{array}$ & $\begin{array}{l}4 \text { Aug } 2009 \\
14 \text { Jul } 2005 \\
3 \text { Aug } 2012 \\
20 \text { Aug } 2010\end{array}$ & 6 exx. \\
\hline 27. Stenurella bifasciata (Müller, 1776) ${ }^{1}$ & EV17: Wysowa-Zdrój & 12 Aug 2006 & $1 \mathrm{ex}$. \\
\hline 28. Stenurella melanura (Linnaeus, 1758) ${ }^{1}$ & $\begin{array}{l}\text { EV29: Bednarka } \\
\text { EV17: Wysowa-Zdrój }\end{array}$ & $\begin{array}{l}4 \text { Aug } 2009 \\
7 \text { Aug } 2008 \\
\text { 8 Jul 2010 } \\
\text { 3-11 Jul } 2011\end{array}$ & 7 exx. \\
\hline 29. Stenurella nigra (Linnaeus, 1758$)^{1}$ & $\begin{array}{l}\text { EA20: Lipinki } \\
\text { EV17: Wysowa-Zdrój }\end{array}$ & $\begin{array}{l}15 \text { Jun } 2013 \\
21 \text { May } 2014 \\
23 \text { Jul } 2010\end{array}$ & 4 exx. \\
\hline Spondylidinae Audinet-Serville, 1832 & & & \\
\hline 30. Spondylis buprestoides (Linnaeus 1758) ${ }^{\mathbf{1}}$ & $\begin{array}{l}\text { EV28: Bartne } \\
\text { EV17: Wysowa-Zdrój }\end{array}$ & $\begin{array}{l}10 \text { Jul } 2013 \\
11-22 \text { Jul } 2005\end{array}$ & 5 exx. \\
\hline 31. Asemum striatum (Linnaeus, 1758) ${ }^{1}$ & EV17: Wysowa-Zdrój & 18 May 2013 & $1 \mathrm{ex}$. \\
\hline 32. Arhopalus rusticus (Linnaeus, 1758$)^{1}$ & $\begin{array}{l}\text { EV17: Blechnarka } \\
\text { EV17: Wysowa-Zdrój }\end{array}$ & $\begin{array}{l}18 \mathrm{Jul} 2007 \\
20 \mathrm{Jul} 2006\end{array}$ & 2 exx. \\
\hline 33. Tetropium castaneum (Linnaeus, 1758$)^{1}$ & $\begin{array}{l}\text { EV17: Blechnarka } \\
\text { EV17: Wysowa-Zdrój }\end{array}$ & $\begin{array}{l}17 \text { Jul } 2007 \\
7 \text { Jul } 2008 \\
18 \text { May 2013: under } \\
\text { bark }\end{array}$ & 4 exx. \\
\hline 34. Tetropium fuscum (Fabricius, 1787) ${ }^{1}$ & $\begin{array}{l}\text { EV18: Małastów1 } \\
\text { EV17: Wysowa-Zdrój }\end{array}$ & $\begin{array}{l}14 \text { Jun } 2013 \\
18 \text { May 2013: under } \\
\text { bark }\end{array}$ & 3 exx. \\
\hline 35. ${ }^{*}$ Tetropium gabrieli (Weise, 1905) ${ }^{\mathbf{1}}$ & EV17: Wysowa-Zdrój & $\begin{array}{l}3 \text { May 2014: under } \\
\text { bark }\end{array}$ & 1 ex. \\
\hline \multicolumn{4}{|l|}{ Cerambycinae Latreille, 1802} \\
\hline 36. Obrium brunneum (Fabricius, 1793) ${ }^{1}$ & $\begin{array}{l}\text { EA20: Bednarka } \\
\text { EV17: Wysowa-Zdrój }\end{array}$ & $\begin{array}{l}16 \text { May } 2013 \\
23 \text { May } 2014 \\
6 \text { May } 2014\end{array}$ & 3 exx. \\
\hline 37. Molorchus minor (Linnaeus, 1758) & $\begin{array}{l}\text { EA20: Bednarka } \\
\text { EA10: Libusza }\end{array}$ & $\begin{array}{l}23 \text { May } 2014 \\
14 \text { Jul } 2004 \\
\text { 12 Jun } 2011\end{array}$ & 6 exx. \\
\hline 38. ! Rosalia alpina (Linnaeus, 1758) ${ }^{\mathbf{1}}$ & $\begin{array}{l}\text { EV17: Blechnarka } \\
\text { EV17: Wysowa-Zdrój }\end{array}$ & $\begin{array}{l}8 \text { Jul } 2007 \\
20 \text { Aug } 2009\end{array}$ & $\begin{array}{l}\text { observ. } \\
2 \text { exx. }\end{array}$ \\
\hline 39. Aromia moschata (Linnaeus, 1758) ${ }^{1}$ & EV17: Wysowa-Zdrój & $\begin{array}{l}12 \text { Jul } 2005 \\
20 \text { Aug } 2010\end{array}$ & 3 exx. \\
\hline 40. Hylotrupes bajulus (Linnaeus, 1758) & $\begin{array}{l}\text { EV17: Blechnarka } \\
\text { EA10: Libusza }\end{array}$ & $\begin{array}{l}24 \text { Jul } 2013 \\
16 \text { Jul } 2005\end{array}$ & 2 exx. \\
\hline 41. ${ }^{*}$ Ropalopus macropus (Germar, 1824) ${ }^{1}$ & EV17: Blechnarka & 14 Jul 2008 & 2 exx. \\
\hline 42. Callidium violaceum (Linnaeus, 1758$)^{1}$ & $\begin{array}{l}\text { EA10: Libusza } \\
\text { EV17: Wysowa-Zdrój }\end{array}$ & $\begin{array}{l}12 \text { Jun } 2011 \\
20 \text { Jun } 2014 \\
11 \text { Jul } 2005\end{array}$ & 6 exx. \\
\hline 43. ${ }^{*}$ Pyrrhidium sanguineum (Linnaeus, 1758$)^{1}$ & $\begin{array}{l}\text { EA20: Lipinki } \\
\text { EV17: Wysowa-Zdrój }\end{array}$ & $\begin{array}{l}13 \text { Jul } 2007 \\
30 \text { Apr } 2014\end{array}$ & 2 exx. \\
\hline
\end{tabular}




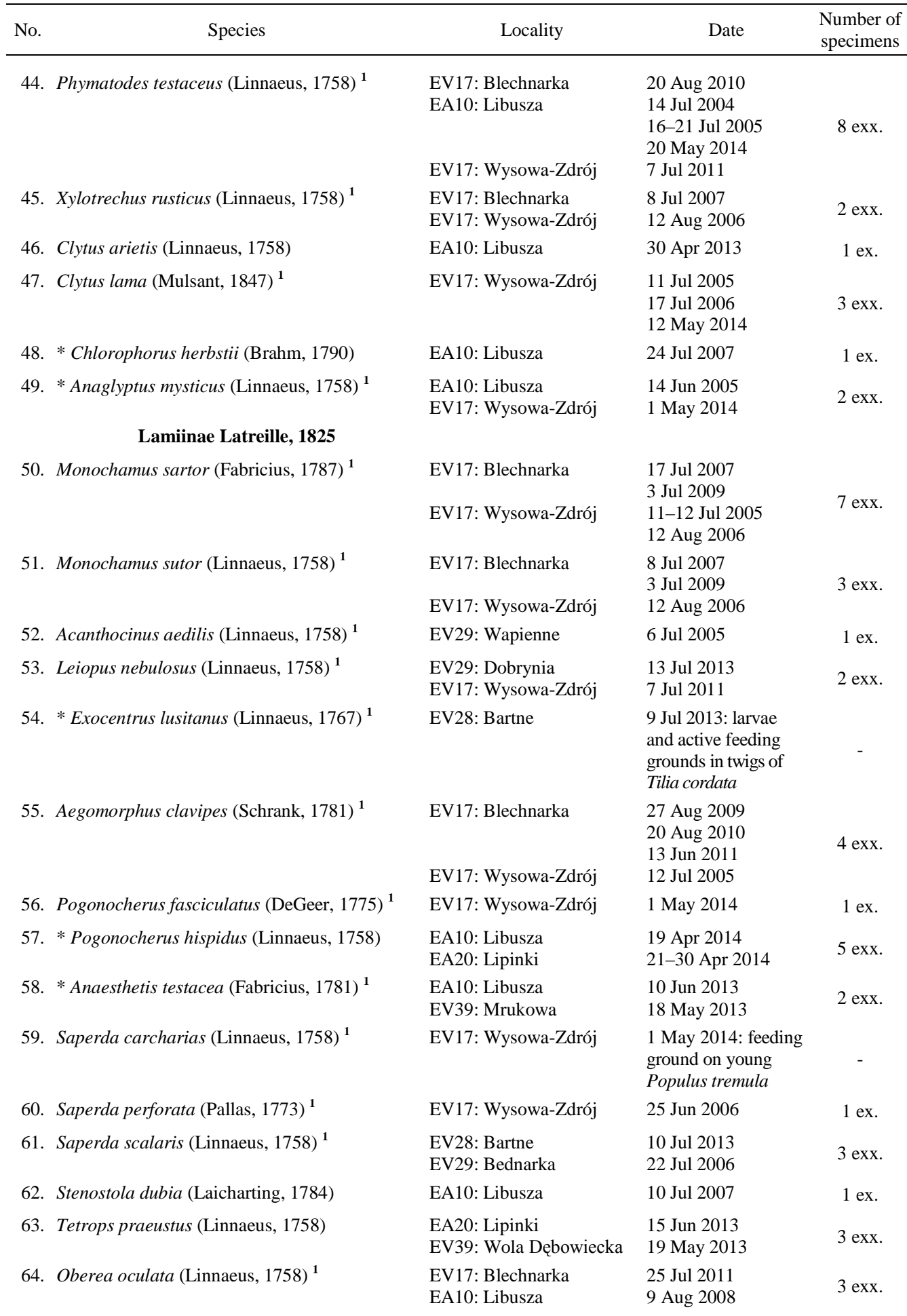




\begin{tabular}{|c|c|c|c|}
\hline Species & Locality & Date & $\begin{array}{l}\text { Number of } \\
\text { specimens }\end{array}$ \\
\hline 65. Oberea erythrocephala (Schrank, 1776) & $\begin{array}{l}\text { EA10: Libusza } \\
\text { EA20: Lipinki } \\
\text { EV39: Nowy Żmigród }\end{array}$ & $\begin{array}{l}\text { 10-16 Jun } 2013 \\
15 \text { Jun } 2013 \\
14 \text { Jul } 2013 \\
19 \text { May } 2013\end{array}$ & 5 exx. \\
\hline 66. Phytoecia affinis (Harrer, 1784) ${ }^{1}$ & $\begin{array}{l}\text { EV28: Bartne } \\
\text { EA10: Libusza } \\
\text { EV17: Wysowa-Zdrój }\end{array}$ & $\begin{array}{l}\text { 10 Jun } 2013 \\
\text { 11 Jul } 2013 \\
\text { 4 Jul } 2004 \\
\text { 14 Jul } 2005 \\
\text { 23 Jun } 2012 \\
\text { 15 May } 2013 \\
\text { 13 Jun } 2011 \\
\text { 17 May } 2013\end{array}$ & 13 exx. \\
\hline 67. * Phytoecia cylindrica (Linnaeus, 1758) & EA20: Bednarka & 16 May 2013 & 2 exx. \\
\hline 68. * Phytoecia nigricornis (Fabricius, 1781) & EV29: Dobrynia & $\begin{array}{l}13 \text { Apr } 2013 \\
16 \text { May } 2013 \\
21 \text { May } 2014\end{array}$ & 7 exx. \\
\hline 69. * Agapanthia intermedia Ganglbauer, $1884^{1}$ & $\begin{array}{l}\text { EV39: Mrukowa } \\
\text { EV39: Nowy Żmigród }\end{array}$ & $\begin{array}{l}18 \text { May } 2013 \\
19 \text { May } 2013\end{array}$ & 3 exx. \\
\hline 70. Agapanthia villosoviridescens (DeGeer, 1775) ${ }^{\mathbf{1}}$ & $\begin{array}{l}\text { EA20: Bednarka } \\
\text { EV17: Blechnarka } \\
\text { EV29: Dobrynia } \\
\text { EA10: Libusza } \\
\text { EV17: Wysowa-Zdrój }\end{array}$ & $\begin{array}{l}\text { 16 May } 2013 \\
\text { 13 Jun } 2011 \\
\text { 13 Jun } 2013 \\
\text { 12 Jun } 2006 \\
\text { 3 Jun } 2010 \\
\text { 20 Jun } 2012 \\
\text { 8 Jul 2010 } \\
\text { 23 May } 2011\end{array}$ & 14 exx. \\
\hline
\end{tabular}

\section{DISCUSSION}

According to summarised literature data, 136 species (70\% of the Polish fauna) of Cerambycidae are currently known from the Eastern Beskid Mountains (Trella 1925, Burakowski et al. 1990, Kubisz et al. 1991, Kubisz \& Hilszczański 1992, Gutowski 1995, Sláma \& Gutowski 1997, Trzeciak 2005, Gutowski et al. 2010, Olbrycht \& Szewkienicz 2013, Olbrycht 2014). However, the occurrence of two species in this region: Stenurella septempunctata (Fabricius 1792) and Stictoleptura fulva (DeGeer, 1775) seems unlikely (Ziarko 1993, Gutowski 1995). The presence of 83 representatives of longhorn beetles has been documented only from the Low Beskids (Kubisz et al. 1991, Kubisz \& Hilszczański 1992, Sláma \& Gutowski 1997, Olbrycht \& Szewkienicz 2013). However the recognition of Anoplodera sexguttata (Fabricius, 1775) as occurring in the Low Beskids from environs of the Gorlice, that is based on the record of Sulma (1931) seems to be doubtful. A. sexguttata is mainly lowland species, associated especially with oaks, while Gorlice city is located a few kilometers north of the border of this region. Therefore, it is more likely that this record refers to a locality from remaining part of the Eastern Beskid Mountains. Including the results of our study the number of cerambycids that have been recorded from this area has increased to 87 species. Furthermore, the number of recorded species is comparable to that of the neighbouring mountain regions. A total of 135 species has been reported in the Western Beskid Mountains (Burakowski et al. 1990, Gutowski 1995, Kozak 2009, Gutowski et al. 2011, Pietraszko \& Warchałowski 2013) and 101 in the Bieszczady Mountains (Nowicki 1858, Śliwiński \& Lessaer 1970, Burakowski et al. 1990, Gutowski 1995, Sláma \& Gutowski 1997, Pawłowski et al. 2000, Holly 2007, Kozak 2010, Kurzawa 2012, Kurzawa et al. 2012, Olbrycht \& Szewkienicz 2013). 
In the Low Beskids, apart from the four new species, the rarest (published the first time by Olbrycht \& Szewkienicz (2013)) species are: Ropalopus macropus, Acanthocinus aedilis, Exocentrus lusitanus and Agapanthia intermedia. It is noteworthy, that the following species, i.e. Stenocorus meridianus, Evodinus clathratus, Nivellia sanguinosa (Fig. 2), Anoplodera rufipes, A. sexguttata, Tetropium gabrieli, Pogonocherus hispidus, Phytoecia cylindrica and $\mathrm{Ph}$. nigricornis were recorded in the Eastern Beskid Mountains only from single observations or have only been confirmed in recent years.

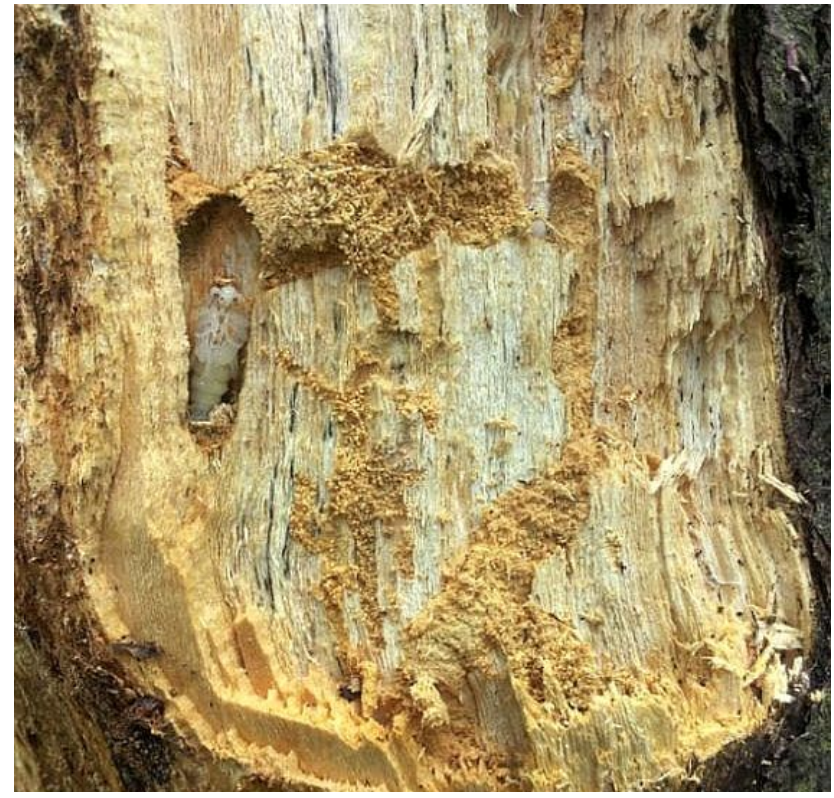

Fig. 2. Pupa and larval feeding grounds of Nivellia sanguinosa (Gyllenhal, 1827) in a Padus avium (Mill.) trunk. Photo by W.T. Szczepański.

Moreover, some records seem to be particularly interesting as, for example, synanthropic Hylotrupes bajulus (which larvae live mainly in wooden elements of building constructions) on a pile of spruce fire-wood next to the forest, at least a few kilometers from the nearest buildings. Similarly, $\mathrm{Ph}$. cylindrica, which usually inhabits open areas, mid-forest clearings and forest edges, was caught in the middle of the hornbeam forest during the sweep-netting of the undergrowth. The presence of $P$. sanguineum in an area, where there are no oaks within a few kilometers, is also noteworthy, because this species prefers oaks as a host plants, and only occasionally chooses other deciduous trees.

At present, the cerambycids' species composition of the Eastern Beskid Mountains and its neighbouring regions appear to be well investigated. However, it is likely that the occurrence of a several next species will be proven still, especially within the Low Beskids. For example, A. rufipes (which was found on the border of the Low Beskids), Chlorophorus herbstii, $P$. hispidus or Ph. nigricornis. Two very rare species in Poland - Macroleptura thoracica Creutzer, 1799 and Stenopterus rufus (Linnaeus, 1767) - were recently reported from the area of the Low Beskids (Olbrycht \& Szewkienicz 2013). In our view, the appearance of central and south European species $S$. rufus in this region may be associated with recent climate changes, that affect many insect species (Tryjanowski et al. 2010). 
Because of the physiographic and geobotanical distinction of the Low Beskids compared to the rest part of the Eastern Beskid Mountains, the authors deem the idea of recognizing this area as a separate zoogeographical region, similar to the one proposed by Tykarski (2011) (the Central Beskid Mountains as a subunit of Beskid Mts. and Foothills), to be reasonable. These differences are emphasized by results of our research, during which 38 species were recorded from only the Low Beskid and 14 only from the remaining part of the Eastern Beskid Mountains. This is also confirmed to a certain extent inter alia taking as an example species of the genus Phytoecia Mulsant, 1839: affinis, cylindrica and nigricornis. The first one is a species that commonly occurs in the mountains (e.g., the Bieszczady Mts.) and one that has been recorded on the strictly montane research plots. The two other species, which are certainly not considered to be mountain elements, have not been found in any of the Low Beskids locations, despite the more intense investigation of the research plots and regular inspections of the local meadows and undergrowth using the sweep-netting method. Two other species that are associated with the beech Fagus L. that appear to confirm this thesis are Rosalia alpina and Stictoleptura tesserula. In our research, all records of these species are located in the Low Beskids or at its border.

\section{ACKNOWLEDGEMENTS}

We are grateful to Professor Adam Ślipiński (CSIRO, Canberra) and another anonymous reviewer for valuable comments and constructive suggestions. We also thank Dr Jolanta Wytwer (MIZ, Warszawa) for her comments and the editorial corrections which significantly improved the original version of our manuscript.

\section{REFERENCES}

Burakowski B., Mroczkowski M. \& Stefańska J. 1990. Chrząszcze - Coleoptera. Cerambycidae i Bruchidae. Katalog Fauny Polski, PWN, Warszawa, 23 (15): 312 pp.

GuTOWsKi J. M. 1995. Kózkowate (Coleoptera: Cerambycidae) wschodniej części Polski. Prace Instytutu Badawczego Leśnictwa, Ser. A, 811: 190 pp.

Gutowski J. M., Hilszczański J., Kubisz D., MiŁkowski M., Mokrzycki T., Plewa R., Przewoźny M. \& WEŁNICKI M. 2010. Distribution and host plants of Leiopus nebulosus (L.) and L. linnei Wallin, Nylander et Kvamme (Coleoptera: Cerambycidae) in Poland and neighbouring countries. Polskie Pismo Entomologiczne 79(3): 271-282.

Gutowski J. M., Piotrowski W. \& RozwaŁka R. 2012. Kózkowate (Coleoptera: Cerambycidae) Poleskiego Parku Narodowego. Parki narodowe i Rezerwaty Przyrody 31 (3): 31-50.

GUTOWSKi J. M., ZiELIŃSKi S. \& BIWO T. 2011. Longhorn beetles (Coleoptera: Cerambycidae) of Romincka Forest. Nature Journal, Opole Scientific Society 44: 145-171.

Holly M. 2007. Nowe stanowiska rzadkich gatunków chrząszczy na terenie Bieszczadzkiego Parku Narodowego oraz w Bieszczadach Zachodnich. Roczniki Bieszczadzkie 15: 243-251.

KONDRACKI J. 2013. Geografia regionalna Polski. Wydawnictwo Naukowe PWN, Warszawa, 440 pp.

KoZAK B. 2009. Nowe stanowisko Cortodera femorata (Fabricius, 1787) (Coleoptera: Cerambycidae) w południowej Polsce. Wiadomości Entomologiczne 28 (4): 279-280.

KozAK B. 2010. Nowe stanowisko Leiopus linnei Wallin, Nylander et Kvamme, 2009 (Coleoptera: Cerambycidae) w Bieszczadach. Wiadomości Entomologiczne 29 (4): 299.

KUBISZ D. \& HILSZCZAŃSKI J. 1992. Fauna kózkowatych (Coleoptera, Cerambycidae) Beskidu Niskiego. Wiadomości Entomologiczne 11 (2): 73-79.

Kubisz D., StOlzmann P. \& GRABowski G. 1991. Owady kambio- i ksylofagiczne rezerwatu „Modrzyna” na Przełęczy Dukielskiej (Beskid Niski). Parki Narodowe i Rezerwaty Przyrody 10: 93-101.

KuRZAWA J. 2012. Distribution of Rhaphuma gracilipes (Faldermann, 1835) (Coleoptera: Cerambycidae) in Europe. Acta Entomologica Silesiana 20: 65-70.

KurZaWA J., SZCZEPAŃSKi W. \& SzCZEPAŃSKi W.T. 2012. Kózkowate (Coleoptera: Cerambycidae) masywu Chryszczatej w Bieszczadach. Acta Entomologica Silesiana 20: 55-64.

LÖBL I. \& SMETANA A. 2010. Catalogue of Palaearctic Coleoptera. Vol. 6: Chrysomeloidea. Apollo Books. Stenstrup, 335 pp.

MAzuR S. 2001. Ryjkowce kserotermiczne Polski: (Coleoptera: Nemonichidae, Attelabidae, Apionidae, Curculionidae). Studium zoogeograficzne. Monografie Fauny Polski 22: 1-378.

OlBRYCHT T. 2014. Kózkowate (Coleoptera: Cerambycidae) obszaru Natura 2000 „Patria nad Odrzechową”. Roczniki Bieszczadzkie 22: 321-327. 
Olbrycht T. \& SzewkiEnicz A. 2013. Kózkowate Coleoptera, Cerambycidae nowe dla Bieszczadów i Beskidu Niskiego. Roczniki Bieszczadzkie 21: 375-380.

PawŁowski J., PetryszaK B., Kubisz D. \& SzwaŁko P. 2000. Chrząszcze (Coleoptera) Bieszczadów Zachodnich. Monografie Bieszczadzkie 8: 9-143.

PietraszKo M. \& WARChAŁOWSKI M. 2013. Pierwsze odkryte ślady żerowania larw Cerambyx cerdo (Linnaeus, 1753) (Coleoptera: Cerambycidae) w Beskidzie Żywieckim. Wiadomości Entomologiczne 32 (2): 153-154.

NowICKI M. 1858. Coleopterologisches über Ostgalizien. Schulnachrichten Jahres-Bericht des Kaiserl. Künigl. OberGymnasiums zu Sambor für das Schuljahr 1858: 1-24.

SlÁmA M. E. F. \& GuTOWSKI J. M. 1997. Xylotrechus capricornis (Gebler, 1830) (Coleoptera: Cerambycidae) gatunek nowy dla polskiej i czeskiej fauny. Wiadomości Entomologiczne 16 (2): 83-97.

Sulma M. 1931. Notatki o Cerambycidach Polski. I. Polskie Pismo Entomologiczne, Lwów, 10: 141-143.

ŠVÁCHA P. \& LAWRENCE J. F. 2014. 2.4. Cerambycidae Latreille, 1802, pp. 77-177. [In:] LESCHEN R. A. B. \& Beutel R. G. (eds.): Handbook of Zoology, Arthropoda: Insecta; Coleoptera, Beetles, Volume 3: Morphology and systematics (Phytophaga). Walter de Gruyter, Berlin/Boston.

ŚLIWIŃSKI Z. \& LESSAER. M. 1970. Materiały do poznania kózek Polski (Coleoptera, Cerambycidae) ze szczególnym uwzględnieniem Bieszczadów Zachodnich. Roczniki Muzeum Górnośląskiego (Przyroda), Bytom 5: 77-127.

TASZAKOWSKI A. 2012. Lądowe pluskwiaki różnoskrzydłe (Hemiptera: Heteroptera) doliny górnej Ropy. Acta entomologica Silesiana 20: 37-54.

Trella T. 1925. Wykaz chrząszczów okolic Przemyśla. Elateridae - Sprężyki, Eucnemidae - Goleńczyki, Cerambycidae - Kózki. Polskie Pismo Entomologiczne 4: 92-96.

Tryjanowski P., PaWlikowski T., PaWlikowski K., BanaszaK-CiBicka W. \& Sparks T. H. 2010. Does climate influence phenological trends in social wasps (Hymenoptera: Vespinae) in Poland? European Journal of Entomology 107: 203-208.

TRZECIAK A. 2005. Nowe stanowisko Axinopalpis gracilis (Krynicki, 1832) (Coleoptera: Cerambycidae) w Polsce. Wiadomości Entomologiczne 24 (1): 53.

TYKARSKI P. 2011. Towards redefining the regional division of Poland for faunistic studies. Polish Journal of Entomology 80: 155-183.

ZIARKO S. 1993. Weryfikacja niektórych błędnych danych dotyczących Cerambycidae (Coleoptera), zawartych w „Katalogu Fauny Polski”. Wiadomości Entomologiczne 12 (1): 15-17.

ZIELIŃSKI S. 2004. Kózkowate (Coleoptera: Cerambycidae) Lasów Mirachowskich na Pojezierzu Kaszubskim. Roczniki Naukowe Polskiego Towarzystwa Ochrony Przyrody „Salamandra” 8: 49-104.

\section{STRESZCZENIE}

\section{[Nowe dane o występowaniu kózkowatych (Coleoptera: Cerambycidae) na terenie Beskidu Wschodniego (Polska)]}

Niniejsza praca prezentuje wyniki badań nad różnorodnością kózkowatych (Coleoptera: Cerambycidae), przeprowadzonych w latach 2004-2014 na terenie Beskidu Wschodniego. Podsumowane zostały również dane literaturowe dotyczące występowania Cerambycidae w Beskidzie Wschodnim (z wyszczególnieniem Beskidu Niskiego), Beskidzie Zachodnim oraz w Bieszczadach. Przedstawiono nowe informacje o występowaniu 70 gatunków z omawianej rodziny chrząszczy, w tym 56 z obszaru Beskidu Niskiego, dla którego podano również 4 nowe gatunki: Pachyta quadrimaculata, Leptura annularis, Pyrrhidium sanguineum oraz Pogonocherus fasciculatus. Łącznie $\mathrm{z}$ nowymi danymi liczba stwierdzonych kózkowatych wynosi: w Beskidzie Wschodnim - 136 gatunków, w samym Beskidzie Niskim - 87 gatunków, w Beskidzie Zachodnim - 135 oraz w Bieszczadach - 101 gatunków. Potwierdzono również obecność kilku rzadko obserwowanych w tej części Polski gatunków: Stenocorus meridianus, Evodinus clathratus, Nivellia sanguinosa, Anoplodera rufipes, A. sexguttata, Tetropium gabrieli, Acanthocinus aedilis, Exocentrus lusitanus, Pogonocherus hispidus, Phytoecia cylindrica, Ph. nigricornis oraz Agapanthia intermedia. Odrębność Beskidu Niskiego jako jednostki zoogeograficznej sugerują liczne różnice w faunie kózkowatych tego obszaru i pozostałej części Beskidu Wschodniego. 